\title{
Adaptation to Real Motion Reveals Direction-selective Interactions between Real and Implied Motion Processing
}

\author{
Jeannette A. M. Lorteije $\mathrm{L}^{1,2}$, J. Leon Kenemans ${ }^{1,2}$, Tjeerd Jellema ${ }^{3}$, \\ Rob H. J. van der Lubbe ${ }^{1,2,4}$, Marjolein W. Lommers ${ }^{2}$, \\ and Richard J. A. van Wezel ${ }^{1,2}$
}

\begin{abstract}
Viewing static pictures of running humans evokes neural activity in the dorsal motion-sensitive cortex. To establish whether this response arises from direction-selective neurons that are also involved in real motion processing, we measured the visually evoked potential to implied motion following adaptation to static or moving random dot patterns. The implied motion response was defined as the difference between evoked potentials to pictures with and without implied motion. Interaction between real and implied motion was found as a modulation of this difference response by the preceding motion adaptation.
\end{abstract}

\section{INTRODUCTION}

Visual motion is processed in specialized areas of the visual cortex, most notably in the mediotemporal and medio-superior-temporal cortical (MT/MST) area, which is part of the dorsal visual pathway (Ungerleider \& Mishkin, 1982). It has been shown that the human MT/MST complex also shows a higher blood oxygenation level dependent signal in response to photographs of objects in motion (e.g., a cup falling off a table or an athlete throwing a ball) than to photographs of the same objects at rest (cup on the table, athlete sitting) (Kourtzi \& Kanwisher, 2000; Senior et al., 2000). This is an interesting finding because form information was generally thought to be processed along the ventral visual pathway (Ungerleider \& Mishkin, 1982) and not in the MT/ MST. A visually evoked potential (VEP) study revealed that this implied motion response is visible as a positive potential at occipital and occipitoparietal electrodes from 260 to $400 \mathrm{msec}$ after stimulus onset (Lorteije et al., 2006). This response is delayed by approximately 100 msec compared to the response to real motion in the same area. These findings suggest that MT/MST

\footnotetext{
${ }^{1}$ Helmholtz Research Institute, The Netherlands, ${ }^{2}$ Utrecht University, The Netherlands, ${ }^{3}$ Hull University, United Kingdom, ${ }^{4}$ Twente University, The Netherlands
}

The amplitude of the implied motion response was significantly reduced after adaptation to motion in the same direction as the implied motion, compared to motion in the opposite direction. At $280 \mathrm{msec}$ after stimulus onset, the average difference in amplitude reduction between opposite and same adapted direction was $0.5 \mu \mathrm{V}$ on an average implied motion amplitude of $2.0 \mu \mathrm{V}$. These results indicate that the response to implied motion arises from direction-selective motion-sensitive neurons. This is consistent with interactions between real and implied motion processing at a neuronal level. receives feedback from higher level visual areas where animate form is analyzed, possibly in temporal cortical areas.

Although these studies show that animate implied motion evokes a response in motion-sensitive areas, it still remains unclear whether the same direction-selective neurons in MT/MST are involved in both real motion and animate implied motion processing. The use of direction-selective adaptation with real motion makes it possible to evaluate whether the implied motion response arises from the same directionally selective neurons that also process real motion.

Numerous studies using different techniques have shown that a prolonged exposure to motion in one direction alters the response of direction-selective neurons to a subsequent motion stimulus in that direction. Single-cell studies in monkeys have shown that most MT neurons are tuned to a specific motion direction (Maunsell \& Newsome, 1987; Zeki, 1978). After exposure to motion in this preferred direction, the cell's response to motion in the same direction is attenuated (Kohn \& Movshon, 2003; Van Wezel \& Britten, 2002). Human psychophysical studies have shown that after being adapted to motion in one direction, the subjects' perceived direction of motion shifts away from the adapted direction (Schrater \& Simoncelli, 1998; Levinson \& Sekuler, 1976). Adaptation to one motion direction can even result in the 
perception of an illusionary motion in the opposite direction, that is, a motion after effect (Mather, Verstraten, \& Anstis, 1998). Furthermore, functional magnetic resonance imaging (fMRI) revealed a decrease in response to motion in the human MT+ complex when it is preceded by motion adaptation in the same direction (Huk, Ress, \& Heeger, 2001). This decrease did not occur when the preceding adaptation occurred in the opposite direction.

Bach and Ullrich (1994) showed that components of the motion VEP can be modulated by motion adaptation, especially a positive peak around 110 to $130 \mathrm{msec}$ after stimulus onset (P1) and a negative peak around 180 msec after stimulus onset (N200). Comparison of motion VEPs with eight different directions deviating from the adapted direction revealed that especially the N200 could be modulated by motion adaptation in a direction-specific manner (Hoffmann, Unsold, \& Bach, 2001); that is, VEPs to motion directions approaching the adaptation direction were much stronger attenuated than VEPs to motion in opposite directions of the adapted direction. In another study with a similar paradigm, it has been shown that the N200 is not only direction specific, but also speed specific (Heinrich, van der Smagt, Bach, \& Hoffmann, 2004). This indicates that this negative response reflects, at least partly, MT/MST activation.

To test the hypotheses that motion-sensitive neurons are also responsive to implied motion and that this interaction is directionally selective, we recorded VEPs to implied motion after three different types of adaptation: (1) a static random dot pattern (RDP), (2) an RDP moving in the same direction as the implied motion, and (3) an RDP moving in the opposite direction as the implied motion. The advantage of this paradigm is that the implied motion test stimuli are always the same; only the preceding adaptation by real motion varies.

In addition to a direction-invariant attenuation of the implied motion response caused by motion adaptation versus static adaptation, we found direction-specific adaptation effects. Most notably, the positive implied motion peak around 280 msec was reduced after motion adaptation in the same direction compared to static adaptation and adaptation in the opposite direction. These results provide evidence that the same motion direction-selective neurons process both real and implied motion.

\section{METHODS}

\section{Subjects}

Twelve women and 11 men participated in this study. All of the participants were naive to the purpose of the study. They had given written informed consent and were paid for expenses. The experiment was conducted in accordance with the declaration of Helsinki (World Medical Association 2000). Recordings from five subjects had to be discarded due to excessive eye movements or noise, leaving data from 10 men and 8 women. These remaining 18 subjects were aged between 19 and 26 years $(22.1 \pm 0.4$ years, mean $\pm S E M)$ and reported normal or corrected-to-normal visual acuity. One man was lefthanded; all other subjects were right-handed.

\section{Stimuli}

Stimuli were presented within a round aperture (radius of $4.3^{\circ}$, black surround) in the middle of the screen. Participants were asked to fixate on a red square $\left(0.2^{\circ} \times\right.$ $0.2^{\circ}$ ) in the center of the screen during trials and to refrain from blinking during the test stimulus.

Trials consisted of an adaptation phase (2000 msec), a variable interstimulus interval (ISI, ranging from 500 to $600 \mathrm{msec}$ ), and a test stimulus (500 msec) (Figure 1). Three types of adaptation were used: a static RDP, a leftward-moving RDP, and a rightward-moving RDP. These different conditions were presented in separate recording blocks to enable top-up adaptation. To ensure strong adaptation at the first trial, every block was preceded by 17 sec of adaptation.

The duty cycle (test duration as a percentage of the total trial duration) of the test stimulus ranged between $16 \%$ and $17 \%$, dependent on the length of the variable ISI. At duty cycles higher than 20\%, test periods containing real motion could cause adaptation effects resulting in an invalid "unadapted" baseline (Bach \& Ullrich, 1994). Although the test periods in the current study did not contain real motion, implied motion may cause similar adaptation effects. However, with this duty cycle the unadapted baseline condition was valid.

In the adaptation phase, participants viewed an RDP that consisted of black and white dots $\left(0.1^{\circ} \times 0.1^{\circ}\right)$ against a gray background. The dot density was $2 \%$ of the total background surface: 1\% white and 1\% black dots. Average luminance of the RDP was $38 \mathrm{~cd} / \mathrm{m}^{2}$. The RDPs either moved coherently to the right or left at $6.8^{\circ} / \mathrm{sec}$, or remained stationary. The final frame of the adapting RDP remained static during the ISI and remained on screen as a static background during the test stimulus. At the onset of the next adaptation period, the RDP was refreshed. Only one sequence of dot motion was shown, in left or right moving order, such that the background for test stimuli preceded by the same direction of adaptation motion was always the same.

The test stimulus consisted of pictures of human agents profiled toward the right or left. The human agents either expressed implied motion (running; Figure 1, top) or did not express implied motion (standing still; Figure 1, bottom). During the test, the RDP remained static. The test contained no real motion, only motion implied by the pictures. These photographs, which were stills from digitally recorded movie clips, showed one of three different persons (two men, one woman). Persons in the photographs were $6^{\circ}$ in height. 
Figure 1. Trials consisted of an adaptation period, a variable ISI, and a test period. During the adaptation period, the RDPs could move to the left or right, or remain static for baseline recordings. However, during the ISI and the test period, the RDP remained static in the background. The test period did not contain any real motion onset, only motion implied by the pictures. The human agents in the (static) test pictures either expressed implied motion (top picture) or did not express implied motion (bottom picture). The running/viewing directions for both implied and implied motionless pictures could be to the left or right. The implied motion response was established as the subtraction VEP of the evoked potentials to pictures with implied motion minus the evoked potentials to pictures without implied motion.

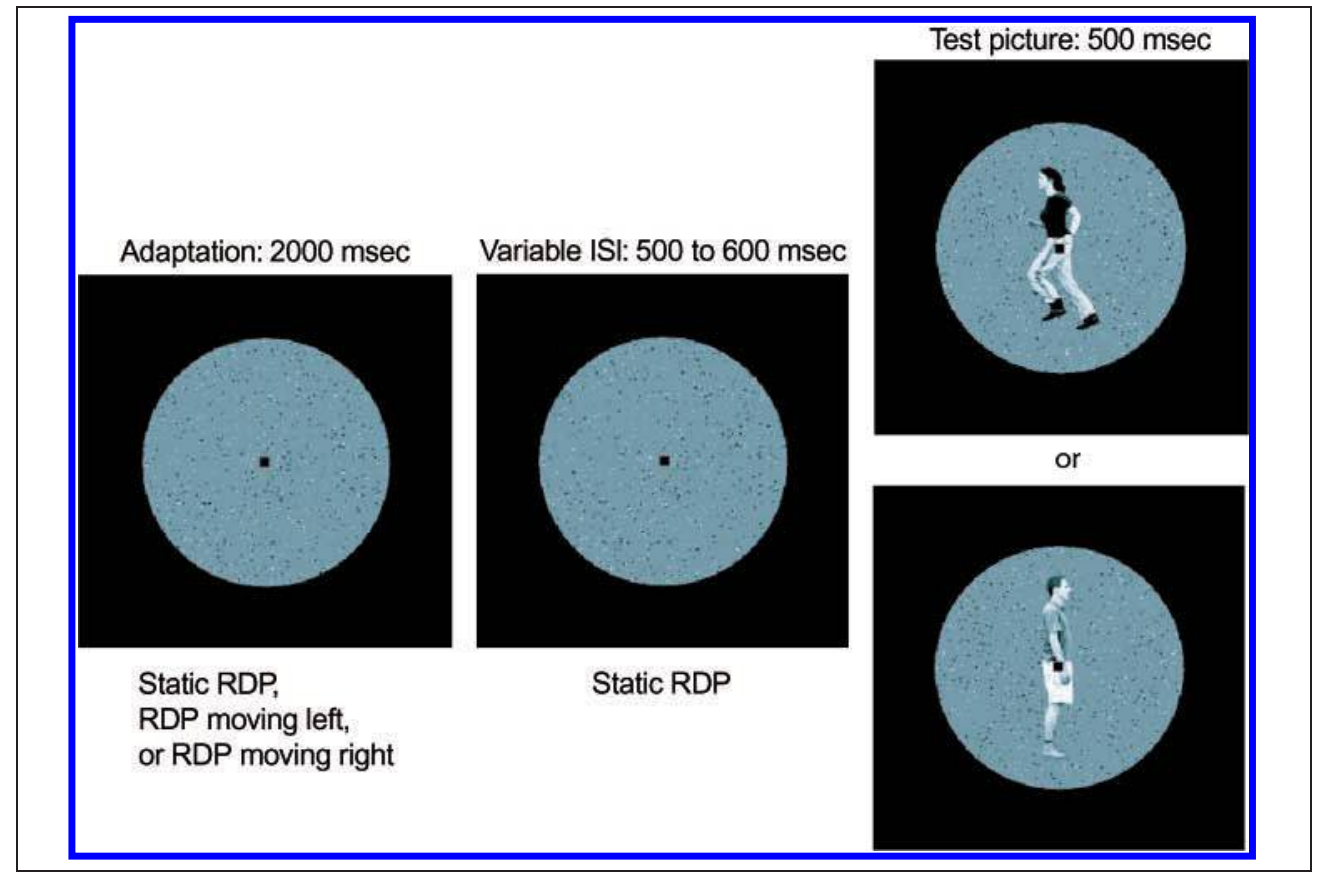

To diminish the influence of luminance on the VEP, the average luminance of the persons (not the background) in the implied motion photographs was adjusted to match that of their implied motionless counterparts.

The difference between the average response to implied motion stimuli minus the average response to stimuli without implied motion was calculated for every subject. This subtraction potential eliminates responses to processes that are common in both conditions, such as stimulus onset or face recognition, and thus mainly reflects the implied motion response. Therefore, whenever we speak about "the implied motion response," we refer to this subtraction potential.

The photographs were presented randomly in both directions. The adapted motion could thus be in the same or opposite direction as the implied motion or as the profiled direction of the images without implied motion. Direction of motion in the RDP and direction of implied motion in the picture were combined in the analysis as same or opposite when they were in the same or opposite direction, respectively. Every condition was presented 240 times.

\section{Experimental Procedure}

Participants were seated in a comfortable chair in a darkened room. They sat in front of a 17-in. computer monitor with a screen resolution of $1024 \times 768$ pixels, $85 \mathrm{~Hz}$, at a viewing distance of $85 \mathrm{~cm}$. The experiment was run in Presentation (Neurobehavioral Systems, Al- bany, CA) and consisted of six blocks of 25 min. Blocks were separated by breaks of at least $3 \mathrm{~min}$ to prevent fatigue and transfer of adaptation from motion blocks onto other blocks. Blocks were presented in a weighted ACBBCA order, with $\mathrm{A}$ and $\mathrm{B}$ being motion adaptation blocks in either left or right direction (varying across subjects) and with $\mathrm{C}$ being stationary adaptation blocks.

A detection task was included to ensure that participants attended to all stimuli. The target stimuli were photographs in which the person was shown from a frontal angle, either running or standing. Participants had to press a button upon detection of a target. The number of targets was $6 \%$ of the total number of trials and they were presented at random. All 18 subjects detected well above $97 \%$ of the attention task stimuli. Five subjects reported a single false alarm, when no target had been presented.

The electroencephalogram (EEG) was recorded from $59 \mathrm{Ag} / \mathrm{Cl}$ ring electrodes, which were mounted in an elastic cap (Braincap, Brain Products, Germany). Scalp electrodes were distributed according to the 10/10 system. Additional electrodes included two electrodes above and below the left eye to record the vertical electrooculogram (EOG), two electrodes on the outer canthi of both eyes for horizontal EOG and one grounding electrode. In addition, two electrodes were applied on both mastoids. Resistance between skin and electrodes was kept below $2 \mathrm{k} \Omega$ throughout the experiment. BrainVision Recorder (Brain Products, Munich, Germany) was used to 
sample and digitize the EEG at $1 \mathrm{kHz}$, and data were filtered (high-pass cutoff at $0.03 \mathrm{~Hz}$, low-pass cutoff at $400 \mathrm{~Hz}$, and a 50-Hz notch filter) and stored on hard disk for off-line analysis. Electrode $\mathrm{Cz}$ was used as reference during the experiment. All scalp electrodes, including $\mathrm{Cz}$, were re-referenced off-line to averaged mastoids for further analysis.

\section{Data Analysis}

BrainVision Analyser (Brain Products) was used for data analysis. The EEG was segmented into stimulus-locked epochs of $600 \mathrm{msec}$ (100 $\mathrm{msec}$ before to $500 \mathrm{msec}$ after stimulus onset). Segments containing an attention task stimulus (or a false response to a test stimulus) and blinks or eye movements (criteria were greater than 100 or less than $-100 \mu \mathrm{V}$ ), or artifacts (criteria were greater than 120 or less than $-120 \mu \mathrm{V}$ on any EEG channel) were excluded from further analysis. VEPs were filtered (high-pass cutoff $0.05 \mathrm{~Hz}$, low-pass cutoff $20 \mathrm{~Hz}, 12 \mathrm{~dB} /$ octave) and baseline corrected (for 100 to $0 \mathrm{msec}$ before stimulus onset). For every subject, the average response per condition was calculated. The difference between the average response to implied motion stimuli minus the average response to stimuli without implied motion stimuli was calculated for every subject.

In an earlier evoked potential study (Lorteije et al., 2006), differences in potential between responses to photographs with and without implied motion were most pronounced at occipitoparietal electrodes. EEG potentials evoked by real motion were clearly visible at the same electrodes (Lorteije et al., 2006; Heinrich et al., 2004; Hoffmann et al., 2001; Bach \& Ullrich, 1994). For this reason, we selected 17 occipital and parietal electrodes for further analysis on peak amplitudes (P7, P5, P3, P1, Pz, P2, P4, P6, P8, PO7, PO3, POz, PO4, PO8, O2, $\mathrm{Oz}$, and $\mathrm{O} 1$; for locations, see N150 in Figure 4A).

\section{Source Analysis}

To locate the neuronal sources underlying the differences in evoked potentials, brain electric source analysis (BESA 2.2; Scherg \& Picton, 1991) was performed on the baseline implied motion grand averages. For each peak, the grand average was calculated over the subjects that were selected for that specific peak in the previous peak amplitude analysis. The analysis included data from all scalp electrodes and not only the 17 posterior ones. BESA modeled location, orientation, and strength of equivalent intracranial dipole sources according to the recorded scalp activity. Grand average peak latencies were established for the P100, the N150, and the P280 as the latencies at which one of the 17 electrodes reached the maximum amplitude for that peak. The optimal dipole solutions were found by searching for a minimum in the residual variance (RV) function at those laten- cies. To reduce the probability of interacting dipoles (i.e., adjacent dipoles with opposing high-amplitude potential fields), the energy constraint of the BESA model was set to 20\% (with the remaining $80 \%$ for the RV criterion), thus favoring source solutions with relatively low dipole strengths (Berg \& Scherg, 1994). Single dipole pairs were used for source models. The location and orientation of the dipoles were bilateral symmetrically constrained.

\section{RESULTS}

\section{Implied Motion VEP}

The focus of analysis was on 17 occipital, temporal, and parietal electrodes that most probably reflected responses from visual motion areas. Three peaks in the implied motion response could be discriminated at these locations (Figure 2): two positive peaks at approximately 100 and 280 msec after test stimulus onset (P100 and a broad P280), and one negative peak around 150 msec after test stimulus onset (N150). Comparison of the implied motion VEPs after the three different adaptation conditions showed that the P100 was attenuated due to motion adaptation (static vs. same and opposite), but this modulation was not dependent on the direction of the motion. In contrast, the P280 was clearly reduced in amplitude after adaptation in the same direction, but not after adaptation in the opposite direction. The negative peak did not show any modulation due to motion.

The amplitude of each peak after static adaptation was calculated for every subject. The amplitude in this static condition had to be large enough to reveal possible modulation in the moving adaptation conditions. Therefore, the maximum of each peak after static adaptation was established for every subject by automatic detection of global maxima for each of the 17 channels in Analyzer. The mean potential over the period of $10 \mathrm{msec}$ before to $10 \mathrm{msec}$ after the maximum was chosen as peak amplitude. Because the scalp distribution varied across subjects (especially due to left- or right-hemisphere dominance), the electrode at which this highest amplitude was found could vary across subjects.

The P100 was established as the peak amplitude between 40 and $120 \mathrm{msec}$ after stimulus onset, for every subject. Likewise, the N150 was located between 100 and $200 \mathrm{msec}$, and the P280 was located within the 240 to $340 \mathrm{msec}$ range. Subjects that had a positive amplitude less than $1.5 \mu \mathrm{V}$ or a negative amplitude greater than $-1.5 \mu \mathrm{V}$ in the static adaptation condition were not included in further analysis for that specific peak.

Using this rule, we included 14 of 18 subjects for the P100 analysis, 18 for the N150, and 15 for the P280. In addition, one subject was excluded for the P280 analysis as she had a much stronger negative peak coinciding in time with the P280 $(-4.9 \mu \mathrm{V}$ at P7 vs. $2.2 \mu \mathrm{V}$ at Pz). 
Figure 2. Grand averages $(n=18)$ of the implied motion response after three types of adaptation at ten electrode positions. Clearly visible are the positive peak around $100 \mathrm{msec}$ (P100), the negative peak around $150 \mathrm{msec}(\mathrm{N} 150)$, and the much wider positive peak around 280 msec (P280) after stimulus onset, which are indicated with arrows for electrode $\mathrm{PO} 3$.

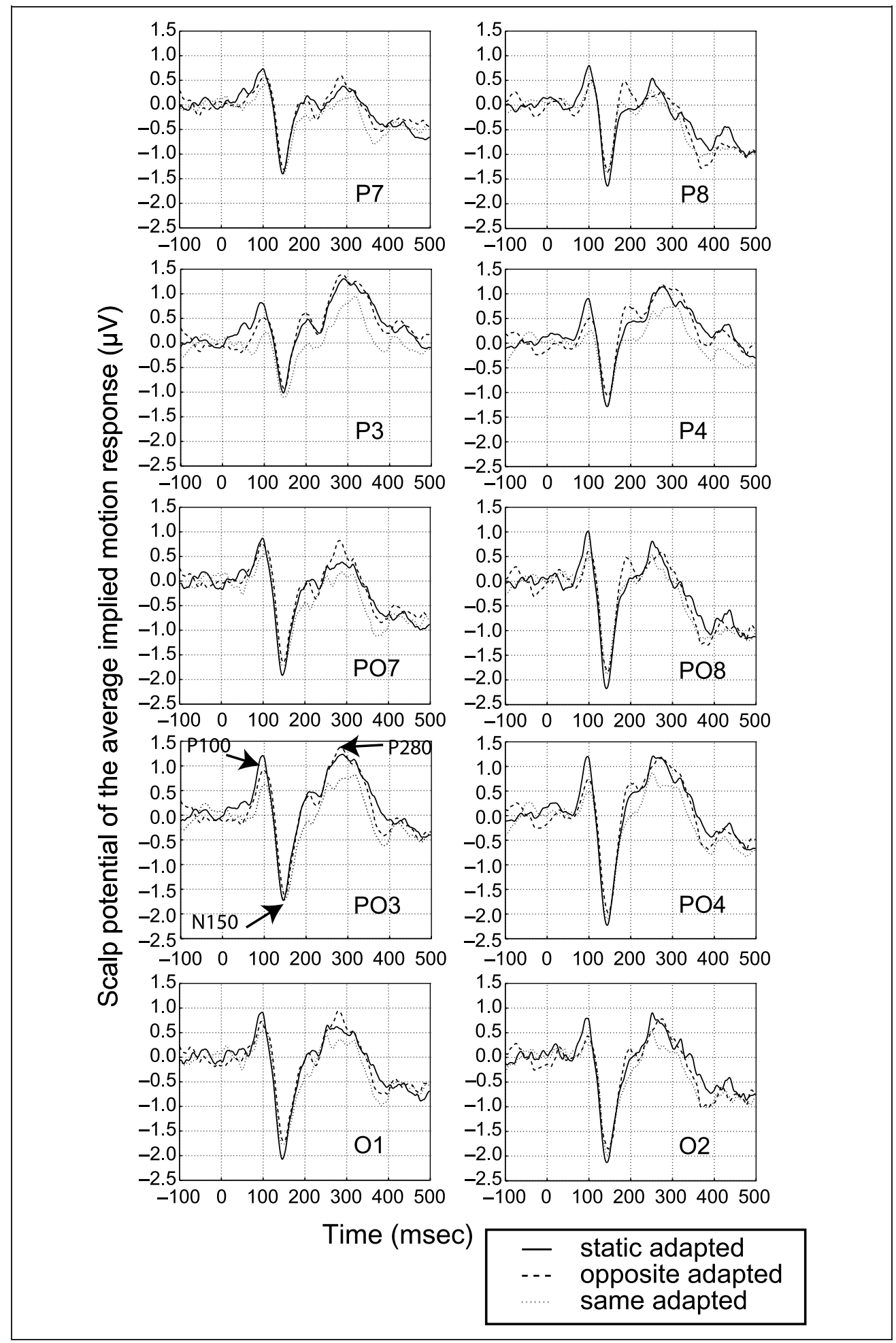

The average maximum (mean \pm SEM) of the P100 was $2.6 \pm 0.2 \mu \mathrm{V}$ for the static adapted condition versus $1.9 \pm 0.2 \mu \mathrm{V}$ for opposite adapted and $1.7 \mu \mathrm{V} \pm 0.3 \mu \mathrm{V}$ for same adapted conditions. The P280 had an average maximum of $2.0 \pm 0.2 \mu \mathrm{V}$ for the static adapted condition, $1.7 \pm 0.3 \mu \mathrm{V}$ for the opposite adapted, and $1.2 \pm$ $0.3 \mu \mathrm{V}$ for the same adapted condition. The average maximum amplitude of the N150 was $-3.3 \pm 0.4 \mu \mathrm{V}$ for static adapted, $-3.2 \pm 0.4 \mu \mathrm{V}$ for opposite adapted, and $-3.3 \pm 0.4 \mu \mathrm{V}$ for same adapted conditions.

\section{Nondirectional Adaptation Effects}

The evoked potentials to implied motion after adaptation to a moving RDP (regardless of direction) were calculated. The amplitudes for these VEPs were obtained 
from the same electrodes that provided the amplitudes for the static RDP condition. A paired $t$ test revealed that the amplitudes of the P100 and P280 after motion adaptation differed significantly from the same peaks after static adaptation ( $p=.000$ and .001 , respectively), whereas the N150 did not change significantly $(p=.628)$.

\section{Direction-specific Adaptation}

To establish direction-specific adaptation effects, the motion adaptation trials were separated into adaptation in the same direction as the implied motion, and adaptation in the opposite direction as the implied motion. The amplitudes of all three peaks after the three adaptation conditions were compared in a repeated measures analysis of variance (ANOVA). Adaptation had a significant effect on the P100 and the P280 (Greenhouse-Geisser corrected $p$ values were .001 and .002 , respectively), but not on the N150 (Greenhouse-Geisser corrected $p$ value was .813). Individual amplitudes after adaptation in the opposite and same direction were normalized by dividing by the corresponding individual amplitudes after static adaptation (Figure 3). Paired $t$ tests revealed that only the amplitude of the P280 was significantly different after adaptation in the same versus opposite direction $(p=.036 ; p=.516$ for $\mathrm{P} 100, p=.261$ for N150).

\section{Scalp Distributions and Source Localization}

To estimate the locations of neural sources underlying implied motion responses, BESA (Berg \& Scherg, 1994; Scherg \& Picton, 1991) was performed on the scalp distribution of the baseline grand averages for the P100 and the P280 peaks (Figure 4A). Source models consisted of single dipole pairs, whose bilateral locations and orientations were mirrored in the midline.

Sources were estimated at the grand average peak latencies, that is, $99 \mathrm{msec}$ for the P100, $145 \mathrm{msec}$ for the N150, and $282 \mathrm{msec}$ for the P280 (Figure 4B). The P100 arose from sources in the occipital lobe. Residual variance (RV, the percentage of scalp data that the model cannot account for; the lower the RV, the better the model fits the data) of this dipole model was only $3.7 \%$.
The sources responsible for the N150 were also located in the occipital lobe, although located slightly more anterior than the P100. RV for the N150 was $1.1 \%$. The dipole pair that fitted the P280 was located even more anteriorly toward the temporal lobe compared to the other peaks, with an RV of $6.8 \%$.

Dipole solutions for the individual data were located in the same way as the grand average sources were established, at the individual peak latencies. However, source locations varied across subjects. The $x, y$, and $z$ positions of the individual source locations were compared in a multivariate test. Because not all participants passed the criteria to be included in analysis for all three peaks $( \pm 1.5 \mu \mathrm{V}$ amplitude), only the source solutions for subjects that were included in analysis for all three peaks were compared statistically. Individual peak locations of 12 subjects were compared in a multivariate analysis, with the three peaks as within-subject factors and the $x, y$, and $z$ positions as measures. Locations of the three peaks did not differ significantly: Hotelling's trace, $F(6,38)=1.1, p=.356$. Univariate tests for the three different coordinates did not reveal significantly different positions for the three peaks either (GreenhouseGeisser corrected $p$ values for $x, y$, and $z$ coordinates were $.49, .17$, and .72 , respectively).

\section{DISCUSSION}

\section{Interactions between Real and Implied Motion}

The current experiment revealed that adaptation to real motion modulated responses to animate implied motion. The modulation consisted of an attenuation of the amplitude of the early component of the evoked potential (P100), which did not depend on the direction of adapting motion, and an attenuation of the later component (P280), which did depend on congruency of adapting motion and implied motion directions. These peaks are in concordance with those found in an earlier study (Lorteije et al., 2006). In that study, dipole source analysis suggested that the later component (P280) originated from motion-sensitive cortex. However, although real and animate implied motion responses appeared to arise from the same cortical area, this does not neces-

Figure 3. Normalized average maximum amplitudes for three typical implied motion peaks after adaptation to real motion in either the same or opposite direction as the implied motion. Paired $t$ tests revealed that only the amplitude of the P280 was significantly different for the two motion adaptation directions.

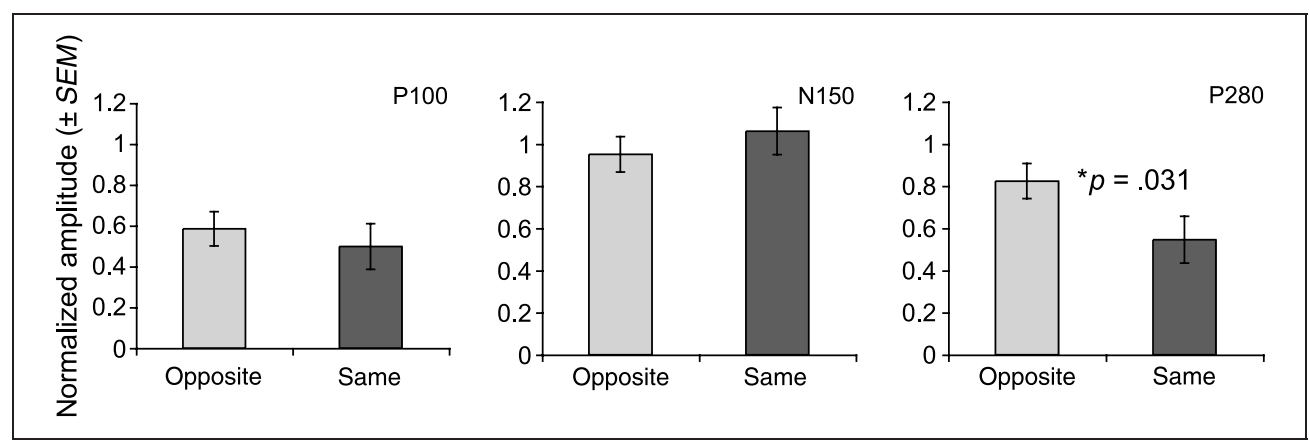




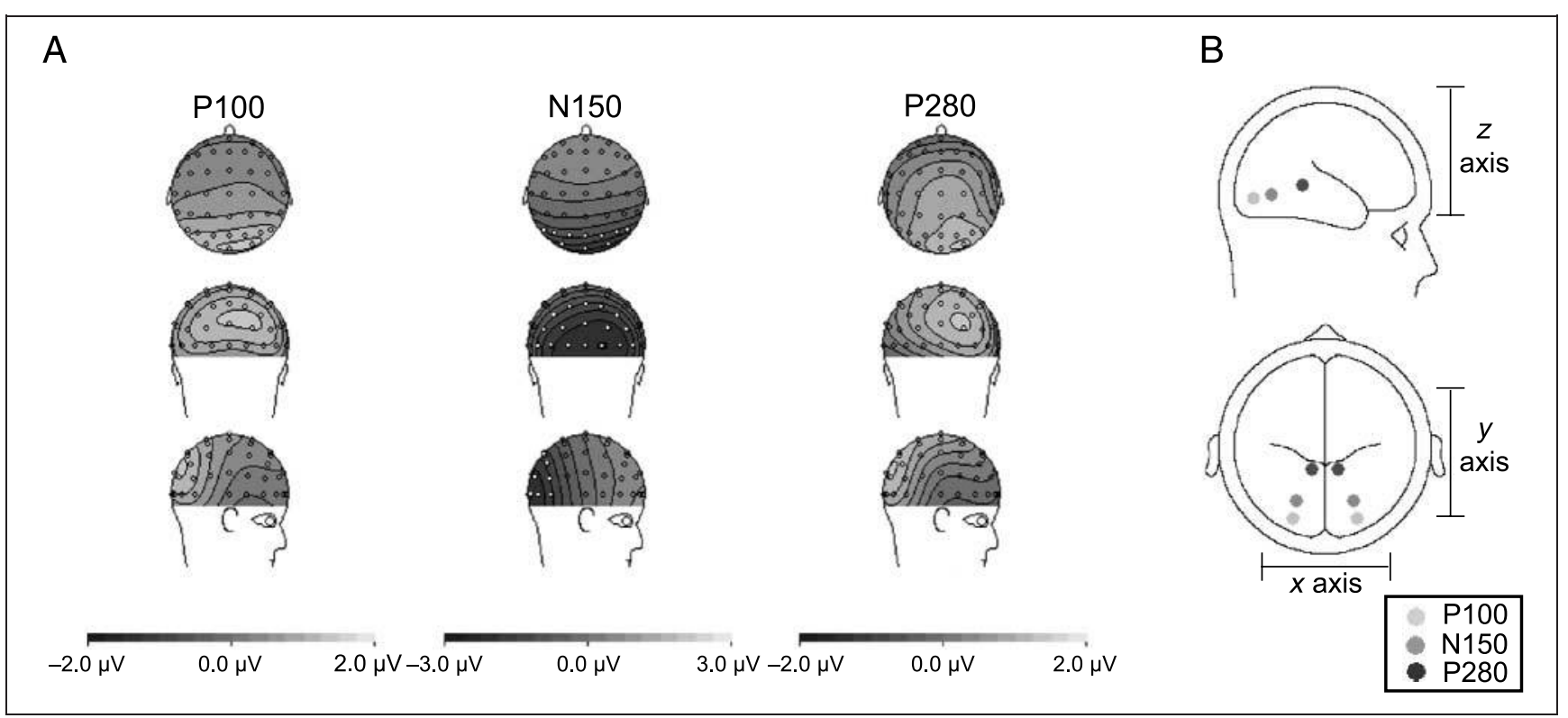

Figure 4. (A) The potential distributions across the scalp of the P100, the N150, and the P280 vary, as is visible in these maps of the grand average implied motion responses at three peak latencies. Note that the scale of the N150 map is larger, as the amplitude of this response was larger than that of both positive peaks. The 17 electrodes that were included in the amplitude analysis are marked with white dots in the N150 maps. (B) Source analysis was performed on the scalp maps. The P100 and N150 are located in the occipital lobe, whereas the P280 seems to be located more anteriorly toward the temporal lobe. However, due to interindividual variation, statistical analysis on the individual source locations revealed that these source locations were not significantly different.

sarily mean that they are processed by the same neurons. Here, for the first time, we have provided evidence that is consistent with interactions at neuronal level between real and animate implied motion processing.

\section{Source Localizations}

Source analysis for the grand average P100, N150, and P280 showed a trend from posterior locations for the P100 toward a more anterior location for the P280, with the N150 in the middle. However, individual locations overlapped and comparison of the source locations for individual subjects did not reveal any significant differences in locations between these peaks. This means that the performed analysis lacked the spatial resolution or statistical strength to discriminate between the three sources. However, the modulation by motion adaptation of the three peaks differed, which indicates that they do arise from different neural populations.

\section{Nondirectional Adaptation}

The amplitude of the P100 peak was attenuated after adaptation with a moving RDP. This modulation is indifferent to the direction of the motion. An earlier study (Lorteije et al., 2006) has hypothesized that this response arises from early visual areas in response to low-level stimulus features such as luminance, spatial frequencies, and orientation. The current results revealed that this peak actually originated from motionor flicker-sensitive neurons, which also responded to nondirectional features of implied motion. The P100 found in the current study coincides in time with the early phase of the P1 component that was found by Di Russo, Martinez, Sereno, Pitzalis, and Hillyard (2001) in their study on early components of the VEP. By combining VEP recordings with fMRI scans, they established that the early phase ( 80 to $110 \mathrm{msec}$ ) of this $\mathrm{P} 1$ arose from the dorsal extrastriate cortex, most probably V3, V3a, and the adjacent middle occipital gyrus. Besides the temporal congruency of the P1 in the Di Russo et al. study and the implied motion P100, the source location found for the latter also resembles that of the former, although the spatial resolution of the current study is low.

\section{Direction-specific Implied Motion Response}

Implied motion typically evoked a positive peak that was maximal around $280 \mathrm{msec}$ after stimulus onset. The amplitude of this peak was significantly attenuated after adaptation to real motion in the same direction as the implied motion, compared to adaptation to real motion in the opposite direction. It has been suggested that this implied motion response is not caused by low-level stimulus differences between the images with implied motion versus images without, such as luminance, orientation, and spatial frequency (Lorteije et al., 2006). In the current paradigm, those features cannot explain the directionally selective adaptation; as the test stimuli were equal for both conditions, only the preceding adaptation direction differed. This strongly suggests that the P280 arises, at least partly, as a response to implied 
motion from motion-sensitive neurons that are directionally tuned. Dipole source analysis of this peak suggests that it arose from an extrastriate area deep between the occipital and temporal lobes, but the spatial resolution was not high enough to reveal the true anatomical source of this response. However, based on the direction-selective motion-sensitive behavior and combined with the implied motion activation found with fMRI (Kourtzi \& Kanwisher, 2000; Senior et al., 2000), MT/MST is a plausible candidate for evoking the P280 implied motion response. Recently, however, Nelissen, Vanduffel, and Orban (2006) defined in macaque monkeys cortical areas rostrally of MT/MST that, like MT/ MST, responded not only to real motion, but also to actions implied in static pictures that MT/MST did not respond to. These areas included the lower superior temporal (LST), the fundus superior temporal region (FST), and the middle of the superior temporal polysensory region (STPm). All three are located near the MT/MST complex and may therefore be part of the human MT/MST complex in previous fMRI studies on implied motion (Kourtzi \& Kanwisher, 2000; Senior et al., 2000). It may thus well be that the P280 arose from human homologues of macaque LST, FST, and STPm instead of MT/MST.

\section{The N150}

The negative peak around $150 \mathrm{msec}$ was not modulated by motion in either direction-selective or directionindependent manner. This suggests that the N150 is a response to implied motion arising from neurons that are not involved in real motion processing. Although this N150 overlaps in time with the N200 evoked by real motion onset, the N150 originates from a different neural population and should not be mistaken for the N200.

Because we established the implied motion response as the difference in response to pictures with implied motion minus pictures without, the N150 may reflect low-level stimulus differences between the two picture conditions irrespective of the presence or absence of implied motion, for example, size, orientation, and spatial frequency. These stimulus features cannot explain the direction-selective P280, but may be responsible for the N150.

In contrast, the P100 that preceded the N150 could be modulated by real motion, that is, its amplitude was reduced after motion adaptation, regardless of direction. Interestingly, this adaptation of an early visual process is not inherited by the process underlying the N150. There are two explanations for this discontinued adaptation. First, it could mean that the neural process that underlay the N150 did not follow the neural process responsible for the P100 in the hierarchy of the visual system. Instead, the N150 might have represented neural processing that was part of a parallel visual path. Second, even when the total response from an earlier process
(P100) was diminished due to adaptation, gain control mechanisms could have enabled further processes (N150) to respond as vigorously as before adaptation by responding to the ratio of the output of the adapted process rather than to the total strength of the output.

\section{Role of the Superior Temporal Sulcus in Implied Motion Processing}

The latency of the implied motion response (240$300 \mathrm{msec}$ ) was longer than that of real motion, which typically has a latency of 150 to $200 \mathrm{msec}$ (Lorteije et al., 2006; Heinrich et al., 2004; Hoffmann et al., 2001; Bach \& Ullrich, 1994). This indicates that implied motion is not processed along the dorsal visual path, as real motion is, but arrives at motion processing areas via a longer route, possibly as a projection from temporal form areas. The implied motion-sensitive VEP contains contributions from motion-sensitive neurons across the complete spectrum of directional selectivity. Therefore, direction-selective adaptation of the VEP is only possible if the feedback input to its underlying generator is also direction-selective.

A good candidate for providing a directionally selective projection is the superior temporal sulcus (STS) region, which seems to be specialized for the perception of bodily actions and postures in both macaque monkeys (Barraclough, Xiao, Oram, \& Perrett, 2006; Jellema \& Perrett, 2003a, 2003b; Jellema, Baker, Wicker, \& Perrett, 2000) and humans (Allison, Puce, \& McCarthy, 2000). Cells in the anterior part of the macaque STS respond to specific articulated body movements and postures, whether executed by an actor, or expected to happen on the basis of the immediately preceding perceptual history (Jellema \& Perrett, 2003b), or when implied by the articulation of limbs in a static body posture (Jellema \& Perrett, 2003a). More importantly, the majority of these cells shows selectivity for the direction of the articulation with respect to the observer, and only a minority will respond to an articulation irrespective of its direction (Jellema \& Perrett, 2006).

Barraclough et al. (2006) showed that neurons in the upper bank, lower bank, and fundus of the rostral STS and inferotemporal cortex are selective to the degree of articulation. Interestingly, neurons that responded stronger to static images of walking persons were more responsive to a movie in which a person was walking forward versus backward, whereas neurons that were more responsive to images of standing postures responded stronger to walking backward versus walking forward.

Thus, populations of STS neurons could provide the information needed for a direction-specific implied motion response in motion-sensitive areas. Evoked potentials arising from the STS responses to faces and objects occur as a negative peak around $170 \mathrm{msec}$ (Itier \& Taylor, 2004a, 2004b). However, it is very unlikely that the N150 found here was STS based. Instead, it arose 
from a much more occipital source. Because similar numbers of STS neurons respond to images with and images without implied motion, the net implied motion response from the STS population would approach zero (Barraclough et al., 2006). This might explain why the STS response was not visible in our EEG recordings.

\section{Shared Neural Structure for Implied and Real Motion}

The direction-specific interaction of implied and real motion processing may play an important role in visual processing. We showed that an image of a person or object expressing implied motion activates directionspecific, motion-sensitive neurons. A strong activation might lead to illusory motion perception. Such a phenomenon has been described by, for example, Freyd (1983) in psychophysical experiments. Freyd showed that observers extrapolate the remembered position of objects according to the implied direction of motion, referred to as representational momentum (RM). When MT/MST is withheld from coherent firing by application of transcranial magnetic stimulation, the RM effect disappears (Senior, Ward, \& David, 2002), which indicates the functional necessity of MT/MST for RM. Our results suggest that rather than this complete disruption of MT/ MST, a more subtle adaptation of direction-selective motion neurons might also eliminate the RM effect.

Note that RM has many forms and can be induced by several stimulus features, including postural cues that were present in the current study, but also by, for instance, an inducing motion sequence (e.g., Freyd \& Finke, 1984). In addition, knowledge of the environment surrounding an object (e.g., gravity, friction) or even semantic cues as inserting inducing words as "bounce" or "crash" causes or modulates RM effects. (For a review on the extensive research that has been performed on RM see Hubbard, 2005.)

The sharing of neural structures for real and implied motion processing is in concurrence with an MEG study by Amorim et al. (2000), which revealed a common substrate in the right centroparietal region for rotational RM and imaginary rotation. These authors found the same scalp distribution for imagining a virtual sea horizon rotate and for the RM effect induced by a rotating virtual sea horizon, which indicates that they are processed in the same cortical area. It would be interesting to use an adaptation paradigm as used in the current article to establish whether those two effects are processed by the same (rotation direction selective), or by different populations of neurons in the same area.

\section{Function of Direction-specific Implied Motion Processing}

A weak activation of motion-sensitive cortex by animate implied motion might not lead to illusory motion, but it could still lower the action potential threshold of neurons with a specific direction tuning, thus making the motionsensitive area more sensitive for motion in the implied motion direction. In natural scenes, a moving animate figure expresses both form cues (implied motion) and real motion cues. The extra activation of motion areas by implied motion would make the viewer more susceptible to the motion of the moving figure, which is especially relevant when the background is also moving. The animate moving figure would thus jump out of the background motion. Furthermore, we often get an intermittent view of a continuous action due to distractions such as occluding objects or blinking of the eyes. To be still able to infer the course of the action in such situations, it would be extremely useful if the fragmented images of articulated body postures would somehow contribute to the action representation and the perceived motion. The direction-selective interaction between real and animate implied motion processing, as described in this study, could well provide for this.

\section{Acknowledgments}

This study was supported by The Helmholtz Research Institute, Utrecht University; the Dutch Science Organization (NWO); the Utrecht University High Potential Program; and the Belgian Science Policy IUAP program. We thank Prof. Dr. A. V. van den Berg for comments and advise on this manuscript.

Reprint requests should be sent to Richard van Wezel, Department of Functional Neurobiology, Utrecht University, Padualaan 8, 3584CH Utrecht, the Netherlands, or via e-mail: R.J.A.vanWezel@ bio.uu.nl.

\section{REFERENCES}

Allison, T., Puce, A., \& McCarthy, G. (2000). Social perception from visual cues: Role of the STS region. Trends in Cognitive Sciences, 4, 267-278.

Amorim, M. A., Lang, W., Lindinger, G., Mayer, D., Deecke, L., \& Berthoz, A. (2000). Modulation of spatial orientation processing by mental imagery instructions: A MEG study of representational momentum. Iournal of Cognitive Neuroscience, 12, 569-582.

Bach, M., \& Ullrich, D. (1994). Motion adaptation governs the shape of motion-evoked cortical potentials. Vision Research, 34, 1541-1547.

Barraclough, N. E., Xiao, D., Oram, M. W., \& Perrett, D. I. (2006). The sensitivity of primate STS neurons to walking sequences and to the degree of articulation in static images. Progress in Brain Research, 154, 135-148.

Berg, P., \& Scherg, M. (1994). Besa version 2.0 handbook. Munich: Megis.

Di Russo, F., Martinez, A., Sereno, M. I., Pitzalis, S., \& Hillyard, S. A. (2001). Cortical sources of the early components of the visual evoked potential. Human Brain Mapping, 15, 95-111.

Freyd, J. J. (1983). The mental representation of movement when static stimuli are viewed. Perception \& Psychophysics 33, 575-581.

Freyd, J. J., \& Finke, R. A. (1984). Representational momentum. Journal of Experimental Psychologv: Learning. Memory. ECognition, 10, 126-132. 
Heinrich, S. P., van der Smagt, M. J., Bach, M., \& Hoffmann, M. B. (2004). Electrophysiological evidence for independent speed channels in human motion processing. Journal of Vision, 4, 469-475.

Hoffmann, M. B., Unsold, A. S., \& Bach, M. (2001). Directional tuning of human motion adaptation as reflected by the motion VEP. Vision Research, 41, 2187-2194.

Hubbard, T. L. (2005). Representational momentum and related displacements in spatial memory: A review of the findings. Psychonomic Bulletin \& Review, 12, 822-851.

Huk, A. C., Ress, D., \& Heeger, D. J. (2001). Neuronal basis of the motion aftereffect reconsidered. Neuron. 32, 161-172.

Itier, R. J., \& Taylor, M. J. (2004a). N170 or N1? Spatiotemporal differences between object and face processing using ERPs. Cerebral Cortex, 14, 132-142.

Itier, R. J., \& Taylor, M. J. (2004b). Source analysis of the N170 to faces and objects. NeuroReport. 15, 1261-1265.

Jellema, T., Baker, C. I., Wicker, B., \& Perrett, D. I. (2000). Neural representation for the perception of the intentionality of actions. Brain and Cognition, 44, 280-302.

Jellema, T., \& Perrett, D. I. (2003a). Cells in monkey STS responsive to articulated body motions and consequent static posture: A case of implied motion? Neuropsvchologia. 41, 1728-1737.

Jellema, T., \& Perrett, D. I. (2003b). Perceptual history influences neural responses to face and body postures. Iournal of Cognitive Neuroscience, 15, 961-971.

Jellema, T., \& Perrett, D. I. (2006). Neural representations of perceived bodily actions using a categorical frame of reference. Neuropsvchologia 44, 1535-1546.

Kohn, A., \& Movshon, J. A. (2003). Neuronal adaptation to visual motion in area MT of the macaque. Neuron, 39, 681-691.

Kourtzi, Z., \& Kanwisher, N. (2000). Activation in human MT/MST by static images with implied motion. Journal of Cognitive Neuroscience, 12, 48-55.

Levinson, E., \& Sekuler, R. (1976). Adaptation alters perceived direction of motion. Vision Research, 16, 779-781.
Lorteije, J. A. M., Kenemans, J. L., Jellema, T., van der Lubbe, R. H. J., de Heer, F., \& van Wezel, R. J. A. (2006). Delayed response to animate implied motion in human motion processing areas. Journal of Cognitive Neuroscience, 18 , $158-168$.

Mather, G., Verstraten, F., \& Anstis, S. (1998). The motion after effect: A modern perspective. Cambridge: MIT Press.

Maunsell, J. H. R., \& Newsome, W. T. (1987). Visual processing in monkey extrastriate cortex. Annual Review of Neuroscience, 10, 363-401.

Nelissen, K., Vanduffel, W., \& Orban, G. A. (2006). Charting the lower superior temporal region, a new motion-sensitive region in monkey superior temporal sulcus. Journal of Neuroscience, 26, 5929-5947.

Scherg, M., \& Picton, T. W. (1991). Separation and identification of event-related potential components by brain electric source analysis. Electroencephalography and Clinical Neurophysiology Supplement, 42, 24-37.

Schrater, P. R., \& Simoncelli, E. P. (1998). Local velocity representation: Evidence from motion adaptation. Vision Research, 38, 3899-3912.

Senior, C., Barnes, J., Giampietro, V., Simmons, A., Bullmore, E. T., Brammer, M., et al. (2000). The functional neuroanatomy of implicit-motion perception or representational momentum. Current Biology, 10, $16-22$.

Senior, C., Ward, J., \& David, A. S. (2002). Representational momentum and the brain: An investigation into the functional necessity of V5/MT. Visual Cognition, 9, 81-92.

Ungerleider, L. G., \& Mishkin, M. (1982). Two cortical visual systems. In D. J. Ingle, M. A. Goodale, \& R. J. W. Mansfield (Eds.), Analysis of visual behavior (pp. 549-586). Cambridge: MIT Press.

Van Wezel, R. J. A., \& Britten, K. H. (2002). Motion adaptation in area MT. Journal of Neurophysiology, 88, 3469-3476.

Zeki, S. M. (1978). Uniformity and diversity of structure and function in rhesus monkey prestriate visual cortex. Journal of Physiology, 277, 273-290. 


\section{This article has been cited by:}

1. Ramona Grzeschik, Martin Böckmann-Barthel, Roland Mühler, Jesko L. Verhey, Michael B. Hoffmann. 2013. Directionspecific adaptation of motion-onset auditory evoked potentials. European Journal of Neuroscience 38:10.1111/ejn.2013.38.issue-4, 2557-2565. [CrossRef]

2. Cosimo Urgesi, Maria Maddalena Savonitto, Franco Fabbro, Salvatore M. Aglioti. 2012. Long- and short-term plastic modeling of action prediction abilities in volleyball. Psychological Research 76, 542-560. [CrossRef]

3. Nick E. Barraclough, Jennifer Ingham, Stephen A. Page. 2012. Dynamics of walking adaptation aftereffects induced in static images of walking actors. Vision Research 59, 1-8. [CrossRef]

4. Jeannette A. M. Lorteije, Nick E. Barraclough, Tjeerd Jellema, Mathijs Raemaekers, Jacob Duijnhouwer, Dengke Xiao, Mike W. Oram, Martin J. M. Lankheet, David I. Perrett, Richard J. A. van Wezel. 2011. Implied Motion Activation in Cortical Area MT Can Be Explained by Visual Low-level Features. Journal of Cognitive Neuroscience 23:6, 1533-1548. [Abstract] [Full Text] [PDF] [PDF Plus]

5. Andrea Pavan, Luigi F. Cuturi, Marcello Maniglia, Clara Casco, Gianluca Campana. 2011. Implied motion from static photographs influences the perceived position of stationary objects. Vision Research 51, 187-194. [CrossRef]

6. C. Urgesi, M. Maieron, A. Avenanti, E. Tidoni, F. Fabbro, S. M. Aglioti. 2010. Simulating the Future of Actions in the Human Corticospinal System. Cerebral Cortex 20, 2511-2521. [CrossRef]

7. Ramona Grzeschik, Martin Böckmann-Barthel, Roland Mühler, Michael B. Hoffmann. 2010. Motion-onset auditory-evoked potentials critically depend on history. Experimental Brain Research 203, 159-168. [CrossRef]

8. Kiyoshi Fujimoto, Akihiro Yagi, Takao Sato. 2009. Strength and variability of the backscroll illusion. Vision Research 49, 759-764. [CrossRef]

9. Zoe Kourtzi, Bart Krekelberg, Richard J.A. van Wezel. 2008. Linking form and motion in the primate brain. Trends in Cognitive Sciences 12, 230-236. [CrossRef]

10. Jeannette A. M. Lorteije, Richard J. A. van Wezel, Maarten J. van der Smagt. 2008. Disentangling neural structures for processing of high- and low-speed visual motion. European Journal of Neuroscience 27, 2341-2353. [CrossRef]

11. Jonathan Winawer, Alexander C. Huk, Lera Boroditsky. 2008. A Motion Aftereffect From Still Photographs Depicting Motion. Psychological Science 19, 276-283. [CrossRef] 